\title{
Zwischen Hamlet, Hanswurst und dem Andrucktermin um 22.30 Uhr - Der Alltag der Theaterkritik ${ }^{1}$
}

\section{Der Kritiker, ein beliebtes "Opfer" der Karikaturisten}

Kaum ein Berufsstand wird so gerne karikiert wie der des Kritikers. Es gibt wunderbare Lithographien von Honoré Daumier zum Thema Kunst-Kritik, entstanden im späten 19. Jahrhundert. Im 20. Jahrhundert war der Theaterkritiker Alfred Kerr ein beliebtes „Opfer“ von Cartoonisten. Und vom Schweizer Dramatiker Friedrich Dürrenmatt gibt es einen Sammelband mit Theaterkritiken aus den 1950er Jahren, enthalten sind darin auch seine Kritiker-Cartoons. Auf einem davon sieht man Kritiker als Läuse im Pelz eines Künstlers. Das ist bezeichnend, wir Kritiker führen ein parasitäres Dasein. Mit diesem Klischee muss ich leben, aber ich lebe ganz gut damit.

Besonders häufig wurde Marcel Reich-Ranicki karikiert. Der Spiegel widmete ihm mehrfach Titelseiten, 1993 konnte man ihn unter der Hauptschlagzeile Der Verreißer sehen - als bissigen Terrier mit zerfetztem Buch zwischen den Reißzähnen. ${ }^{2} 1995$ folgte die berühmt gewordene Fotomontage mit Reich-Ranicki, der den Grass-Roman Ein weites Feld zerreißt. ${ }^{3}$

${ }^{1}$ Dieser Beitrag basiert auf dem gleichlautenden Vortrag, den der Verf. im Sommersemester 2018 im Rahmen der Ringvorlesung Ästhetische Kritik im Philosophischen Seminar der Universität Heidelberg gehalten hat.

2 Der Spiegel. Heft 40, 1993.

3 Der Spiegel. Heft 34, 1995. 
Soviel zu gezeichneten Karikaturen. Auf der Bühne selbst findet man ebenfalls Kritiker-Karikaturen, recht viele sogar. Ein sehr altes Beispiel bilden die Handwerker-Szenen aus Shakespeares Sommernachtstraum (1595/96). Die Figuren um den Handwerker Zettel kritisieren sich bei ihren Theater-Proben ständig gegenseitig. Im fünften Akt führen sie dann ihren Schwank Pyramus und Thisbe auf, und das reizt sofort den kritischen Geist der Zuschauer. Die Bühnenfigur Hippolyta sagt über die Inszenierung innerhalb der Inszenierung: „Dies ist das einfältigste Zeug, das ich jemals hörte.“ Aber es wird auch gelobt. Mit den Worten „Gut gebrüllt, Löwe!“ feiert die Figur des Demetrius den extrovertierten Darsteller des Löwen. Ein vergiftetes Lob, das zum geflügelten Wort im Büchmann wurde.

Sehr bekannt ist auch die Kritiker-Karikatur des Sixtus Beckmesser aus Richard Wagners Oper Die Meistersinger von Nürnberg (1867). Beckmesser ist der Prototyp des Pedanten. Angeblich soll sich Richard Wagners Kritiker Eduard Hanslick in Beckmesser wiedererkannt haben.

In der Saison 2017/18 stand die Krimiklamotte Arsen und Spitzenhäubchen (1941) des Amerikaners Joseph Kesselring auf dem Spielplan des Heidelberger Theaters. In diesem Stück wird ein Kritiker als „Hanswurst" entlarvt, er heißt Mortimer und ist eine äußerst fragwürdige Figur. Im Zentrum der Komödie stehen die beiden mörderischen Schwestern Abbey und Martha Brewster, aus purem Mitleid befördern die schrulligen Alten vereinsamte ältere Herren ins Jenseits. Der Plot mutet plump an, wenn man aber bedenkt, dass zur Entstehungszeit des Stücks in Nazi-Deutschland Euthanasie-Programme zum systematischen Massenmord an Kranken und Behinderten durchgeführt wurden, bekommt Kesselrings schwarze Komödie über eine Mordserie aus angeblichem Mitleid eine ganz andere Dimension.

Die Figur des Kritikers ist ein Neffe der mörderischen Alten. Mortimer nimmt seine Profession nicht ernst. Er gibt zu, die von ihm besuchten Vorstellungen schon nach dem ersten Akt zu verlassen, um anschließend bösartige Verrisse zu schreiben. In Heidelberg empörte sich der Schauspieler Hans Fleischmann über das mangelnde Berufsethos Mortimers. Fleischmann hob zu einer wutschnaubenden Philippika gegen die Theaterkritik an. Die Kritiker seien Dilettanten, sie hätten keinerlei Ahnung von den existenziellen Nöten eines Schauspielers. Dieser Wutanfall steht so nicht bei Kesselring, er wurde in den Abend montiert. Tatsächlich stammt er aus der Horrorkomödie Theater des Grauens, einem Film von 1973. ${ }^{4}$ Vin-

${ }^{4}$ Originaltitel Theatre of Blood, Großbritannien 1973, Regie: Douglas Hickox, Drehbuch: Anthony Greville-Bell, Stanley Mann u. John Kohn. 
cent Price spielt darin einen eitlen Schauspieler, der all seine Kritiker nach der Anleitung von Shakespeare-Stücken umbringt. Dabei denkt man unwillkürlich an den Goethe-Vers ,Schlagt ihn tot, den Hund! Es ist ein Rezensent“ aus dem 1774 geschriebenen Gedicht Rezensent, das Reich-Ranicki in seiner Frankfurter Anthologie als das dümmste aller Goethe-Gedichte geißelte. ${ }^{5}$ Einspruch, Euer Ehren: Es ist nicht das dümmste aller Goethe-Gedichte: Reich-Ranicki übersah völlig den ironischen Gehalt der Verse.

Im weiteren Verlauf der Vorstellung wurde Arsen und Spitzenhäubchen nach allen Regeln der Dekonstruktion durch die inszenatorische Mangel gedreht - die Krimikomödie wandelt sich zur Backstage-Comedy. Diese Form der Dekonstruktion wurde zweieinhalb Jahrzehnte lang an Frank Castorfs Berliner Volksbühne erprobt. Dass Castorfs Regie-Handschrift die gesamte Inszenierung von Arsen und Spitzenhäubchen prägte, war kein Zufall, denn der Regisseur des Abends hieß Milan Peschel, ein prominenter Filmschauspieler, der viele Jahre lang bei Castorf an der Volksbühne gespielt hatte (zur Dekonstruktion kommen wir später noch einmal kurz zurück).

Besonders humorvoll gelungen ist die Kritiker-Karikatur Ego aus dem Animationsfilm Ratatouille. ${ }^{6}$ Ego - nomen est omen - ist zwar ein Restaurant-Kritiker und kein Theaterkritiker, aber seine Wesenszüge treffen all die Klischees, die man mit diesem Berufsbild verbindet. Diese Klischees lauten in etwa so: Der Kritiker ist maßlos eitel; er ist besserwisserisch; er liebt sein Metier nicht; er ist ein gescheiterter Künstler, der sich für seinen Dilettantismus an den echten Talenten rächt; und schlimmer noch: er spreizt sich auf impertinente Weise maliziös.

Am Ende von Ratatouille wird Ego aber richtig sympathisch, er bekennt sich zur Hausmannskost namens Ratatouille. Der Gemüseeintopf weckt in ihm sentimentale Kindheitserinnerungen. Man kann diese Filmszene auch dahingehend interpretieren, dass hochgezüchtete Kulinarik und ästhetische Finessen nicht überschätzt werden sollten. Das Einfache und Deftige verschaffe den wahren Genuss. Das klingt ein bisschen populistisch, es wird hier aber auf charmante Weise gezeigt.

Ratatouille wurde 2007 in den USA gedreht, ein Jahr später mit dem Oscar dekoriert. Laut einer BBC-Umfrage von 2016 gehört der Film zu den 100 bedeutendsten Streifen des 21. Jahrhunderts. Er steht zwar „nur“ auf Platz 93, aber immerhin. Wobei die Anmerkung erlaubt sei:

${ }^{5}$ Frankfurter Allgemeine Zeitung, 20.01.1990.

${ }^{6}$ Ratatoille, USA 2007, Regie: Brad Bird u. Jan Pinkawa, Drehbuch: Jim Capobianco, Emily Cook, Kathy Greenberg u. Bob Peterson. 
Solche Bestenlisten, die auch zum Aufgabenfeld von Kritikern gehören, sind immer fragwürdig. Auch im Theater kennt man solche Rankings. ${ }^{7}$

Es gibt noch viel mehr Kritiker-Karikaturen, hier noch ein Beispiel aus dem Bereich der Realsatire. Im November 1995 wurde im Schauspielhaus Bochum das Stück Die Lügen der Papageien uraufgeführt, geschrieben wurde es von Andreas Marber. Der damalige Bochumer Intendant Leander Haußmann brachte es auf die Bühne. Den konkreten Anlass bildete ein Stuttgarter Skandal: Anfang der 1990er Jahre wurde das Feuilleton der Stuttgarter Nachrichten von Klaus B. Harms geleitet, einem sehr angesehenen Kollegen.

Klaus B. Harms fühlte sich dazu berufen, die Seiten zu wechseln. Er tat dies entweder aus maßloser Naivität oder aus purer Eitelkeit. Jedenfalls schrieb er ein Stück und reichte das Manuskript zur freundlichen Prüfung im Staatstheater Stuttgart ein. Doch wie sollte sich der Stuttgarter Intendant Friedrich Schirmer positionieren, wie seine Dramaturgie? Ablehnen und damit riskieren, langfristig dem kritischen Groll des Stuttgarter Feuilletonchefs ausgesetzt zu sein? Oder sollte die Theaterleitung das Stück zur Uraufführung annehmen und sich damit selbst dem Vorwurf der Korrumpierbarkeit aussetzen? Ein unlösbarer Fall.

Friedrich Schirmer trat die Flucht nach vorne an und machte den Skandal öffentlich. Daraufhin verlor der Kritikerkollege seinen Posten als Feuilletonchef der Stuttgarter Nachrichten - und der Dramatiker Andreas Marber nahm die Ereignisse zum Anlass, Die Lügen der Papageien zu schreiben. Bei der Bochumer Uraufführung saß fast die gesamte deutsche Kritiker-Zunft im Parkett und konnte miterleben, wie sie selbst durch den Kakao gezogen wurde, namentlich.

All diese Beispiele zeigen: Theaterkritiker sind eigentlich erbärmliche Kreaturen, zumindest aus der Sicht betroffener Künstler. Doch wo bleibt - frei nach Erich Kästner - das Positive? Es gibt tatsächlich eine sympathische Kritiker-Karikatur. Sie trägt die Züge von Benjamin Henrichs, der bis vor ein paar Jahren für die Zeit und die Süddeutsche Zeitung geschrieben hat. Benjamin Henrichs erschien in einer Inszenierung von Thomas Bernhards Dramoletten, und zwar als Kritiker-Puppe. Geschrieben hatte Thomas Bernhard seine Dramolette zu den Intendantenwechseln von Claus Peymann, sie tragen den Sammeltitel Claus Peymannn kauft sich eine Hose und geht mit mir essen. Philipp Tiedemann hatte sie vor rund 20 Jahren am Wiener Burgtheater inszeniert. In einem der Dramolette packt die Bühnenfigur Peymann einen Applauskoffer, um dieses Gepäckstück von einer Intendanz zur nächsten mitnehmen zu

${ }^{7}$ Etwa die jährlichen Kritikerumfragen der Fachzeitschriften theater heute und Die deutsche Bühne. 


\section{Fünf exemplarische Kritikerpersönlichkeiten: Lessing, Börne, Fontane, Kerr und Luft}

Normalerweise heißt es, die Theaterkritik sei ein Kind der Aufklärung und habe mit Gotthold Ephraim Lessings Hamburgische Dramaturgie in der zweiten Hälfte des 18. Jahrhunderts begonnen. So steht es in Manfred Braunecks Theaterlexikon und Wikipedia plappert es munter nach. Ich möchte dieser These widersprechen: Die Theaterkritik ist so alt wie das Theater selbst.

Aus der griechischen Antike sind uns zwar keine Kritiken nach dem heutigen Verständnis dieser publizistischen Form überliefert, aber die Poetik des Aristoteles hätte ohne ein kritisches Bewusstsein für die dramatische Kunst niemals entstehen können. Seine Poetik beschäftigt sich vorrangig mit der Wirkungsästhetik der Dramen - dazu gehören folgende Stichworte: Furcht, Schrecken und Katharsis. Außerdem propagierte Aristoteles die Einheit der Handlung. Als weiteren Beleg für das enorme Alter der Theaterkritik - zumindest in mündlicher Form - möchte ich die griechischen Dionysien erwähnen. Diese kultischen Theaterwettbewerbe sind ohne ein kritisches Bewusstsein nicht denkbar. Um einen Preisträger küren zu können, muss zuvor ein Prozess der kritischen Abwägung stattgefunden haben. Die ersten Belege für solche Wettbewerbe datieren auf das Jahr 534 vor Christus. Der älteste bekannte und noch heute gespielte Preisträger heißt Aischylos. Halten wir also fest: Die Theaterkritik existiert seit rund 2500 Jahren.

Die publizistische Form als gedruckter, gesendeter oder online verbreiteter Meinungsbeitrag über eine Premiere ist freilich sehr viel jünger. Diese Form beginnt in der Tat mit Lessing und dem Versuch, in Hamburg ein deutsches Nationaltheater zu etablieren - ein Theater mit literarischem, dramaturgischem und darstellerischem Anspruch. Das Projekt scheiterte relativ bald, aber es hatte eine nachhaltige Wirkung - etwa auf Dalberg und Schiller in Mannheim oder auf Goethes Weimarer Hoftheater. Beide Häuser, das in Mannheim und das in Weimar, heißen bis heute Nationaltheater.

Bei Lessings Hamburgische Dramaturgie handelt es sich um eine Kompilation kritisch-analytischer Texte, die hauptsächlich zum theaterinternen Gebrauch bestimmt waren. Wie soll geschrieben, wie inszeniert und wie gespielt werden? - Darum ging es. Und es ging darum, wie sich ein 
deutsches Nationaltheater von der französischen Klassik emanzipieren kann. Lessing polemisierte kräftig gegen die Franzosen. Im Gegenzug brach er eine Lanze für Shakespeare und für ein Theater bürgerlicher Prägung. Entstanden ist die Hamburgische Dramaturgie zwischen 1767 und 1769. Anfangs kritisierte Lessing noch seine Schauspieler, aber die Ensemblemitglieder waren äußerst empfindlich. Deshalb konzentrierte sich Lessing bald nur noch auf rein formale Aspekte der Theaterliteratur und der Theaterpraxis.

Springen wir von Lessing weiter ins frühe 19. Jahrhundert $\mathrm{zu}$ einem ganz anderen Charakter, zu Ludwig Börne (1786-1837), dem großen Polemiker und politisch orientierten Kritiker. Seine Texte gehören zum weiten Feld der operativen Publizistik. Theater- und Literaturkritik waren für Börne ein Vehikel dafür, massiv auf seine Zeit und auf die beengten Verhältnisse während der Ära von Metternich einzuwirken. Er hatte ständig mit den Anfeindungen der Zensur zu kämpfen. Mit seinen Schriften wurde Börne zum Idol des Jungen Deutschland. Anfangs war er auch fast so etwas wie eine literarische Vaterfigur des elf Jahre jüngeren Heinrich Heine (1797-1856). Doch ein Vater-Sohn-Verhältnis, man weiß es, knirscht manchmal gewaltig. So auch bei den beiden nach Paris emigrierten Schriftstellern. Sogar noch nach Börnes Tod polemisierte Heine gegen den Kollegen in der literarischen Kampfschrift über Ludwig Börne (1840). Börnes kritischer Lakonismus, vor allem aber seine polemische Schärfe wirken mustergültig. Norbert Oellers wies in einer stilistischen Betrachtung der Börne-Kritiken ausdrücklich auf ihren mündlichen Duktus hin. ${ }^{8}$

Der Leser wird durch Börnes radikale Subjektivität dazu angeregt, einen eigenen Standpunkt zu entwickeln - auch in Opposition zum Verfasser. Kritiken sind nicht dazu da, einen Konsens herzustellen, sie sollen einen Diskurs anregen. Insofern sind sie dem dialektischen Prinzip verpflichtet. Noch wichtiger vielleicht als Börnes sprachliche Brillanz ist die Tatsache, dass er seine Kritiken mit dem Hier und Jetzt verband. Der viel gescholtene Elfenbeinturm war nicht sein Platz, sondern er vertrat seine politisch-kritische Zeitgenossenschaft mit Nachdruck. Wohl am besten gelungen ist ihm dies in seinen mehrbändigen Briefen aus $\mathrm{Pa}$ ris - unverhüllte, subjektive Reportagen über das Frankreich nach der Julirevolution von 1830 .

Gehen wir jetzt weiter in unserer kleinen Kritikerparade zu Theodor Fontane (1819-1898). Sein Geld hat Fontane hauptsächlich als Journalist verdient, erst spät als Romancier. Er war Kriegsberichterstatter, war England-Korrespondent, und als Wanderer durch die Mark Branden-

\footnotetext{
${ }^{8}$ OelLers (1979), S. 175.
} 
burg pflegte er das Genre des Reise-Feuilletons. Als Fontane bereits 51 war, wurde er für hundert Taler im Monat Theaterkritiker der Vossischen Zeitung. In der Fontane-Werkausgabe füllen die Theaterkritiken von 1870 bis 1894 vier Bände. Durch seine Texte werden die von ihm besuchten Theaterabende sinnlich nachvollziehbar. „Meine Force ist die Schilderung“, sagte er zu seiner kritischen Methode. ${ }^{9}$ Auch er pflegte den Sprachwitz, das aber in gefälliger, unprätentiöser Form. Der Hang zum Mündlichen, zum Plauderton ist - wie bei Ludwig Börne - unverkennbar. Das unterstreicht auch der Titel der ersten Kritikensammlung, herausgegeben von Paul Schlenther wenige Jahre nach dem Tod Fontanes. Der Band hieß Kritische Causerien über Theater (1905).

Fontane präsentiert sich als Kritiker durch und durch subjektiv, er schreibt ungeniert aus der Ich-Perspektive. Klar in der Diktion ist er auch bei Verrissen: „Es ist ein unerquickliches Machwerk von Grund aus“, urteilte er etwa über Karl Gutzkows Spätwerk Der Gefangene von Metz. ${ }^{10}$ Geradezu revolutionär positionierte er sich im Alter, als er eine Lanze für den jungen Gerhart Hauptmann brach. Fontanes Kritik über Hauptmanns Vor Sonnenaufgang war ein Fanal. Stück und Kritik markieren einen Epochenwandel, mit beiden beginnt 1889 der Naturalismus in Deutschland. Bei der Premiere herrschten tumultähnliche Zustände im Parkett: Sozialdemokratische Gedanken auf der Bühne, heftige Szenen des Alkoholismus, die Problematik von Erbkrankheiten, ein Vergewaltigungsversuch, ein Selbstmord, dazu die krassen seelischen Nöte, die eine Totgeburt mit sich bringt - in diesem Stück gibt es keine Helden mehr, nur noch Opfer. Damals eine höchst explosive Mischung, aber Fontane schrieb eine Eloge: Hauptmann habe „nicht bloß den rechten Ton“ getroffen, „er hat auch den rechten Mut - und zu dem rechten Muthe die rechte Kunst. Es ist töricht, in naturalistischen Derbheiten immer Kunstlosigkeit $\mathrm{zu}$ vermuten. Im Gegenteil, richtig angewandt (worüber denn freilich zu streiten bleibt) sind sie ein Beweis höchster Kunst." ${ }^{\text {"11 }}$

Fontane vertrat eine nonchalante Haltung gegenüber dem kritischen Metier. Es gäbe keine Regeln für das Schreiben von Kritiken, behauptete er in der Besprechung des Lustspiels Die Staatskunst der Frauen (1877) von Felix Dahn. Fontane beschreibt den Stückinhalt akkurat, er vergleicht das Werk mit Shakespeares Falstaff, mokiert sich gleich zu Beginn, dass sich das Publikum zu reserviert verhalten habe und widmet am Ende zwei Absätze den Schauspieler-Leistungen. Keine Zeile zur In-

${ }^{9}$ So Fontane am 14.02.1854 in einem Brief an Theodor Storm, zitiert nach: RosT, Wolfgang E. (2013): Örtlichkeit und Schauplatz in Fontanes Werken. Berlin: de Gruyter, S. 67.

10 Fontane (1979), Erster Band, S. 21

11 Fontane (1979), Vierter Band, S. 204. 
szenierung, nichts zum Bühnengeschehen, nichts über das Bühnenbild. Dafür zwei knappe, entscheidende Sätze über die Kritik an sich:

Je länger man das kritische Metier treibt, je mehr überzeugt man sich davon, daß es mit den Prinzipien und einem Paragraphen-Codex nicht geht. Man muß sich auf seine unmittelbare Empfindung verlassen können. ${ }^{12}$

Ja - ich stimme zu, es gibt kein Regelwerk für das Schreiben von Theaterkritiken. Jeder Abend stellt neue Herausforderungen. Jeder Abend verlangt Offenheit für Neues. Auch davon schreibt Fontane in diesem Text.

1894 verfasste Fontane seine letzte Kritik, ein Jahr zuvor schrieb Alfred Kerr seine erste in Berlin. Er gilt als einflussreichster Theaterkritiker des 20. Jahrhunderts. In seiner Hauptmann-Verehrung übernahm er die Stafette von Fontane. Der alte Kritiker ebnete dem jungen Kollegen sogar den Weg, indem er sich für ihn bei den Herausgebern des Magazins für Literatur aussprach. Dazu äußerte sich Fontane mit folgenden Worten:

Ich lese immer mit Vergnügen, was Ihr Mitarbeiter Dr. Kerr im ,Magazin' schreibt. Heute hat er ganz besonders ins Schwarze getroffen, und Sie müssen mir gestatten, da ich Dr. Kerr nicht kenne, Ihnen dies auszusprechen. Es ist das weitaus Beste, was über Wildenbruch je gesagt worden ist. ${ }^{13}$

Ein Ritterschlag für den 1867 in Breslau geborenen Sohn eines jüdischen Kaufmanns. Kerr konnte gewaltig grollen, aber auch überschwänglich loben, wenn ihm ein Theaterabend besonders gut gefiel - so wie Hauptmanns Biberpelz, der 1919 im Theater an der Königgrätzer Straße in Berlin inszeniert wurde. Kerrs mit römischen Ziffern gegliederter Text beginnt mit einem hymnischen Stakkato in zwei Absätzen:

Ein wahres Fest. Keinen besseren Abend hat vermutlich dieser bald abgeschlossene Bühnenwinter gesehen. Von Anfang bis Schluß ein herzhaftes Gelächter. Bis die Stühle wackeln. ${ }^{14}$

Expressive Sätze wie diese schleuderte Kerr gern in die publizistische Landschaft. Entweder wirkten sie wie ein Blitz. Oder der Kritiker verbreitete Weihrauch um ein Kunstereignis. Das verdeutlichte der Grafiker Karl Rössing 1928 auf seinem Holzschnitt Der Kritiker. Diese Karikatur zeigt unverkennbar die Gesichtszüge Alfred Kerrs. Seine Schreibfeder

\footnotetext{
12 Fontane (1979), Zweiter Band, S. 93.

13 VIETOR-ENGLÄNDER (2016), S. 63.

14 Kerr (1998), S. 789.
} 
sieht aus wie der Blitz von Gottvater-Zeus, in der anderen Hand hält er das Weihrauchfässchen. Der Sessel gleicht dem Thron des Kritikerpapstes, und in den beiden Ziffernkolumnen dahinter kann man dreierlei erkennen: romanische Kirchenfenster, aber auch die mosaischen Gesetzestafeln und nicht zuletzt Kerrs Marotte, seine Kritiken mit römischen Ziffern zu gliedern. Für den Kritiker Kerr stellt der Text ein visuelles Phänomen dar - und dieses Phänomen dient der Selbststilisierung. Der Leser musste keine Zeile lesen, nicht einmal den Autorennamen, konnte aber anhand der römischen Ziffern erkennen: Hier schreibt Kerr. So grenzte er sich vom medialen Kontext ab und gab sich eine herausgehobene Position.

Sein stilistisches Credo verdichtete Kerr 1917 zu der Formel „Dein Ausdrucksziel: das Knappere. / Der Inhalt: Aude sapere!"15 - Kurz und knackig soll's sein, zugleich gedanklich anspruchsvoll. Außerdem proklamierte Kerr, die Kritik gehöre zur Dichtkunst - gleichrangig neben Lyrik, Dramatik und Epik. ${ }^{16}$ Eine streitbare Position.

Kerr scheute sich vor nichts und niemandem. Er ätzte gegen den Polizeipräsidenten genauso wie gegen den Kaiser. Besonders gerne attackierte er Kritikerkollegen wie Karl Kraus, Herbert Jhering oder Siegfried Jacobsohn, den er genüsslich des Plagiats bezichtigte. Nicht einmal vor Schiller hatte der Kritiker Respekt. Über dessen Fiesco polterte er 1908: „Schiller, es geht nicht. Man kann nicht einen tückisch klugen Helden zeichnen, der so ein Blödian ist.“"17 Aber Kerr konnte sich auch irren, er unterschätzte beispielsweise Bertolt Brechts Bedeutung.

Nach jahrzehntelanger Treue zu Hauptman kam 1933 der jähe Bruch, da sich der Dramatiker nicht öffentlich vom NS-Regime distanziert hatte. Kerr verfasste daraufhin eine bittere Kampfschrift gegen sein einstiges Idol. Die Nazis verbrannten Kerrs Schriften auf dem Berliner Opernplatz, er selbst musste emigrieren. Bald darauf wurde in Deutschland die Kritik gänzlich verboten, per Dekret von Goebbels im November 1936. Die Kritik galt als zersetzend und als Indiz einer ,jüdischen Kunstüberfremdung“. Sie wurde in den gleichgeschalteten Medien durch den so genannten „Kunstbericht“ ersetzt. Kritiker durfte es nicht mehr geben, nur noch so genannte „Kunstschriftsteller“. Bertolt Brecht reagierte darauf mit seinem Gedicht Verbot der Theaterkritik. ${ }^{18}$

${ }^{15}$ Kerrs Motto zur Einleitung zu den Gesammelten Schriften, zitiert nach KeRR (1972), S. 3.

16 A.a.O, S. 4.

17 Kerr (1985), S. 41.

18 Im Rahmen der Svendborger Gedichte, vgl. BRECHT, Bertolt (1997): Gedichte I. Bd. 3 der Ausgewählten Werke in sechs Bänden. Frankfurt am Main: Suhrkamp, S. 343f. 
Als diese Verordnung in Kraft trat, war Kerr bereits seit fast drei Jahren im Exil - unter extrem prekären Bedingungen. 1948 kehrte Kerr zu einer Vortragsreise nach Deutschland zurück. Im Hamburger Hotel Atlantic erlitt er einen Schlaganfall und war daraufhin halbseitig gelähmt. In der Nacht zum 12. Oktober 1948 schied er freiwillig aus dem Leben.

Ist die Kritik eine eigene Kunstgattung, wie von Kerr proklamiert? Ich habe da meine Zweifel. Lieber halte ich mich an Friedrich Luft (1911-1990), die Stimme der Kritik im RIAS. Diese Sendung wurde von 1946 bis kurz vor Friedrich Lufts Tod Ende 1990 Sonntag für Sonntag im RIAS ausgestrahlt. 15 Minuten Theaterkritik ohne jeden O-Ton, ohne Interview-Partner. Nur Lufts Stimme war zu hören. Von 11.45 Uhr bis 12 Uhr. Die Stimme der Kritik gilt bis als heute die längste Live-Serie der Rundfunkgeschichte.

Friedrich Luft sprach gehetzt, oft hüstelnd. Durch sein kritisches Hopplahopp unterschied er sich vom sonst so sonoren Sound der Sprecher. Luft wurde rasch sehr populär bis weit in die DDR hinein, wo der RIAS auch gehört wurde. Jede Sendung endete mit der Floskel „Gleiche Welle, gleiche Stelle“, mit der Friedrich Luft auf die nächste Stimme der Kritik hinwies. Er stellte sich nicht erhaben über seine Hörer, sondern streute in seine Viertelstunde über das Theater alltagssprachliche Floskeln genauso ein wie sehr spezielle Termini aus der Welt des Theaters. Diese Begriffe präsentierte er aber so, dass sie jeder verstand, auch der Laie, der höchst selten oder gar nicht ins Theater geht. Schon in seiner allerersten Stimme der Kritik im Februar 1946 wurde er zur Identifikationsfigur. Luft beschrieb sich als Hungerleider unter Hungerleidern:

Luft ist mein Name. Friedrich Luft. Ich bin 1,86 groß, dunkelblond, wiege 122 Pfund, habe Deutsch, Englisch, Geschichte und Kunst studiert, bin geboren im Jahre 1911, bin theaterbesessen und kinofreudig und beziehe Lebensmittel der Stufe II. $\mathrm{Zu}$ allem trage ich neben dem letzten Anzug, den ich aus dem Krieg gerettet habe, eine Hornbrille. ${ }^{19}$

Der Hörfunk war Lufts wichtigstes Medium. Aber während seines gesamten Berufslebens schrieb er auch für Zeitungen. Sehr früh schon in der Neuen Zeitung, deren Feuilleton von Erich Kästner geleitet wurde. Später war Luft Chefkritiker der Welt und der Berliner Morgenpost. Seine Zeitungstexte wirken wie protokollierte gesprochene Sprache. Während meiner ersten Redakteursjahre in Berlin durfte ich Lufts Texte redigieren und ins Blatt „heben“. Diese Erfahrung hat mich bis heute geprägt. Nicht so sehr in stilistischer Hinsicht. Ich wollte Lufts gehetzte Diktion und seine teils ungewöhnliche Wortwahl nie kopieren. Imponiert hat mir

${ }^{19}$ LufT (2018), S. 11. 
aber sein pragmatisches Berufsethos. Er sah seine Rolle darin, als „spezialisierter Reporter“ aus den Theatern zu berichten, nicht als Künstler wie Alfred Kerr. Den Mut zur Meinung und zum ästhetischen Urteil schließt das nicht aus. Diese Haltung ist mir sympathisch, für mich stellt die Kritik eine journalistische Dienstleistung dar. Nicht mehr und nicht weniger. Vorbildlich ist für mich auch, dass Luft stets Distanz zum Theaterbetrieb wahrte. Er wolle mit den Künstlern nicht „panebratsch“ sein, wie er sagte. Eine typische Luft-Vokabel: „panebratsch“. Ich hatte sie vorher nie gehört. Sie stammt aus dem Ostpreußischen. Luft meinte damit, dass er sich nicht vereinnahmen lassen wolle. Er ging nie zu Premierenfeiern. Von seiner eigenen Person machte Luft - trotz seines hohen Bekanntheitsgrades - nie viel Aufhebens, im Gegenteil, er pflegte ein gewisses britisches Understatement. Vielleicht eine Prägung durch seine schottische Verwandtschaft.

Nun habe ich fünf prominente männliche Persönlichkeiten der Theaterkritik vorgestellt: Lessing, Börne, Fontane, Kerr und Luft. Das ist natürlich vollkommen unzulänglich. Kein einziges Wort bisher über eine Kritikerin. Deshalb möchte ich mit Nachdruck darauf hinweisen, dass in den literarischen Salons des späten 18. und des frühen 19. Jahrhunderts hervorragende Frauen agierten, lauter hochbegabte Moderatorinnen des kulturellen Diskurses: Rahel Varnhagen, Bettina von Arnim oder Henriette Herz. Letztere übte einen enormen Einfluss auf den noch sehr jungen Ludwig Börne aus. Und wenn man sich heute in den FeuilletonRedaktionen umschaut, fallen einem so versierte Kritikerinnen ein wie Christine Dössel bei der Süddeutsche Zeitung, wie Claudia Burckhardt bei theater heute oder wie Irene Bazinger bei der FAZ auf. Zu den Mitbegründerinnen des immer wichtiger werdenden Online-Forums nachtkritik.de gehört mit Petra Kohse eine ehemalige taz-Redakteurin. Sie hat eine hervorragende Dissertation über Friedrich Luft verfasst.

Wen habe ich in meiner kleinen Kritiker-Parade rein aus Platzgründen verdrängt? - Sehr viele: Otto Brahm, Alfred Polgar, Kurt Tucholsky, Henning Rischbieter, Sibylle Wirsing, Günther Rühle, Georg Hensel, Sigrid Löffler oder den brillanten Stilisten Gerhard Stadelmaier. Ja, vor allem Stadelmaier. Die Reihe ließe sich noch lange fortsetzen.

\section{Andrucktermin 22.30 Uhr - Zur Aktualität der Theaterkritik}

Opern- und Schauspielkritiken werden in den deutschen Tageszeitungen so aktuell wie möglich publiziert - das heißt zwei Kalendertage nach der Premiere, anders geht es nicht wegen der abendlichen An- 
drucktermine. In Zeiten von Twitter, WhatsApp \& Co. erscheint uns das extrem langsam. Nicht wenige Zuschauer teilen über diese Kanäle schon während der Pause mit, ob ihnen der Abend gefällt oder nicht. Diese spontanen Statements sind zwar selten begründet, aber sie können das Meinungsklima beeinflussen.

Die schnellsten professionellen Kritiken hört man im Radio oder man liest sie auf speziellen Online-Plattformen. Hervorragendes leisten die Kollegen der Sendung Fazit im Deutschlandradio. Sie beginnt kurz nach 23 Uhr, dauert knapp eine Stunde und wird nach Mitternacht vom Deutschlandfunk wiederholt. Dauern Premieren besonders lange, müssen die Radio-Kritiker die Vorstellungen vorzeitig verlassen. Ihre Livegespräche mit den Moderatoren sind zwar improvisiert, sie wirken aber authentisch und fundiert.

Ein paar Stunden langsamer als Fazit sind die Online-Portale von Die Deutschen Bühne und nachtkritik.de. Der Name sagt es bereits: Geschrieben wird in der Nacht, die Kritiken müssen bis 7 Uhr morgens an die Redaktion von nachtkritik.de gemailt werden, ab 10 Uhr sind die Texte $\mathrm{zu}$ lesen. Als nachtkritik.de vor zwölf Jahren gegründet wurde, machte sich die Branche darüber lustig. Heute wird das Onlineforum sehr ernst genommen - zu Recht. 30 bis 50 Kritiken werden pro Monat online veröffentlicht. nachtkritik.de beschränkt sich auf Schauspiel-Kritiken, während Die Deutsche Bühne auch Opern- und Tanztheater-Kritiken online publiziert. Beide Foren bevorzugen Kritiken über Ur- und Erstaufführungen oder ungewöhnliche Regie-Handschriften. Das StandardRepertoire ist ihnen nicht so wichtig.

Aber nochmals zu den Feuilletons der Tageszeitungen: Erstaunlicherweise haben hier Opern- und Schauspielkritiken Priorität. Sie werden rasch gedruckt und landen kaum im Stehsatz. Anders verhält es sich bei Buchkritiken, die erst Wochen oder gar Monate nach dem Erscheinen der Titel rezensiert werden. Auch Ausstellungskritiken werden nur selten am Tag der Ausstellungseröffnung oder unmittelbar danach publiziert.

Bei der darstellenden Kunst hingegen pochen die Redaktionen auf eine größtmögliche Aktualität - wie gesagt: Das heißt im Printbereich zwei Tage nach dem Ereignis. Am Morgen nach der Premiere wird geschrieben, anschließend redigiert, schließlich gedruckt. Parallel wird auch das E-Paper im Netz zugänglich gemacht. Überregionale Zeitungen werden normalerweise gegen $18 \mathrm{Uhr}$ angedruckt, bei Regionalzeitungen wie der Rhein-Neckar-Zeitung läuft die Rotation gegen 22.30 Uhr an. Im Extremfall, bei einer Bundestagswahl oder während der Fußballweltmeisterschaft, kann es auch später werden. Dann muss der Vertrieb mit zusätzlichen Spediteuren neu organisiert werden, damit die gedruckten 
Exemplare auch im entferntesten Winkel des Verbreitungsgebiets bis etwa sechs Uhr am Morgen im Briefkasten landen.

Geht es bei Theaterkritiken im Printbereich aber nicht doch schneller? Partiell ja. Neuerdings werden von einer ganzen Reihe von Zeitungsverlagen die E-Paper-Ausgaben schon am Vorabend des jeweiligen Erscheinungstages online zur Verfügung gestellt. Es gab aber schon in der Weimarer Zeit die schnelle Form der Pausenkritik, die am Morgen nach der Premiere auf Seite 1 zu lesen war. Schon Alfred Kerr hat das praktiziert: drei, vier Sätze, per Telefon diktiert, Umfang ca. 15 bis 20 Druckzeilen. In München und Berlin waren solche Pausenkritiken noch bis etwa zur Jahrtausendwende üblich. Ich selbst habe von Fall zu Fall auch welche geschrieben. Besonders gut in Erinnerung ist mir eine Pausenkritik von Heiner Müllers Hamlet-Inszenierung am Deutschen Theater Berlin. Müller hatte mit den Proben schon lange vor der Maueröffnung im Herbst 1989 begonnen, die Premiere erfolgte im März 1990. Dieser sehr spezielle Hamlet wurde zum Endzeit-Spektakel der DDR. Müller verknüpfte die Shakespeare-Tragödie mit seiner düsteren, postdramatischen Hamletmaschine. Hier war nicht nur etwas faul im Staate Dänemark, hier wurde auch der Fäulnisprozess der Deutschen Demokratischen Republik vor Augen geführt. Der Abend dauerte acht Stunden. Beginn 16 Uhr, Ende gegen Mitternacht. Während der ersten Pause sollte ich eine Pausenkritik für Seite 1 schreiben. E-Mails konnte man damals noch nicht verschicken, Handys gab es auch noch nicht, und die wenigen Telefonleitungen zwischen Ost- und West-Berlin waren völlig überlastet. Per Fahrrad habe ich es aber geschafft, während der etwa einstündigen ersten Pause vom Deutschen Theater in Ost-Berlin durch eines der provisorischen Mauerschlupflöcher zur Redaktion zu radeln. Schnell 20 Zeilen getippt, danach wieder retour zu Hamlet. Ich war noch pünktlich und konnte anderntags die ausführliche Kritik schreiben. Solche Pausenkritiken sind heute aus der Mode gekommen, die E-Paper-Ausgaben im Internet sind einfach schneller.

\section{Der Stellenwert der Theaterkritik - Debatten-Feuilleton versus Rezensionsfriedhof}

Machen wir uns nichts vor, Feuilleton-Seiten oder Kultursendungen sind keine Garanten für hohe Quoten. Das waren sie noch nie. Aber sie waren stets ein Gradmesser für die Qualität von Medien, und sie richten sich an die Multiplikatoren der Gesellschaft. Die Lese- oder Einschaltquote kultureller Inhalte liegt, je nach Thema, zwischen drei und 15 Prozent. 
Die Kritik selbst ist ein Bestandteil des Feuilletons, sie dominiert das Ressort aber nicht. Andere journalistische Formate wie Interviews, Berichte, Nachrufe, Laudationes, Reportagen, Kommentare oder Glossen kommen hinzu - Beiträge über gesellschaftliche Debatten nicht zu vergessen, aktuell beispielsweise über die \#MeToo-Debatte.

Es gab eine Zeit, da waren Kritiken regelrecht verpönt. Standen zu viele auf einer Seite, sprach man vom „Rezensionsfriedhof“. Gepflegt wurde dagegen das Debatten-Feuilleton. In den 1980er Jahren begann dieser Trend mit dem Historikerstreit. Protagonisten waren Jürgen Habermas und Ernst Nolte, geführt wurde ihre Debatte über die Singularität des Holocaust in der Zeit und in der FAZ. Danach folgten Debatten über Gentechnik und Ethik, über den Turbokapitalismus, über die Gentrifizierung oder die Fluchtursachen. Vieles davon hat Frank Schirrmacher in der $F A Z$ initiiert. Nun läuft die nächste große Debatte über Chancen und Risiken der Künstlichen Intelligenz.

Die Kritik kann sich in diesem Umfeld trotzdem noch behaupten. In Die Zeit finden Sie mindestens eine Theaterkritik pro Woche, in der $F A Z$ sind es durchschnittlich drei bis fünf. Im Spiegel finden sich nur noch selten Theaterkritiken. In Regionalzeitungen hingegen, zumindest solchen mit einem ernst zu nehmenden Kulturteil, werden alle wichtigen Premieren des Verbreitungsgebiets besprochen. Hinzu kommen ausgewählte Highlights der Theater-Bundesliga. Vollständigkeit schafft kein einziges Medium, weder Print, noch Funk, noch online. Das ist kein Wunder angesichts der Zahlen. Laut der Werkestatistik 2015/16 des Deutschen Bühnenvereins wurden in der vorletzten Saison 5115 Werke in 8346 unterschiedlichen Inszenierungen im deutschsprachigen Raum gezeigt. 4700 davon waren Premieren, der Rest Wiederaufnahmen.

\section{v. Neue Formenvielfalt als Herausforderung}

Für den Schauspiel-Kritiker gibt es längst keinen verlässlichen Kanon mehr. Die Formenvielfalt ist enorm - und sie wächst immer weiter. Schreib-, Spiel- und Inszenierungsmoden kommen und gehen. Gut so, variatio delectat - Abwechslung gefällt.

Zurzeit geraten gerade die so genannten „Textflächen“ aus der Mode. Das sind postdramatische Formen, wie sie Elfriede Jelinek pflegt. Klare Rollenprofile gibt es darin nicht mehr, sondern nur noch Stimmungsbilder, Betrachtungen und Kommentare in Prosaform. Für Regisseure stellen diese Textflächen eine enorme Herausforderung dar. Viele begegnen ihnen mit skandierenden Chören wie im antiken Theater. Einar Schleef 
hat das bei der Uraufführung von Elfriede Jelineks Sportstück am Wiener Burgtheater geradezu mustergültig vorgemacht.

Immer noch „up to date“ ist die Dekonstruktion klassischer Dramen oder adaptierter Roman- und Film-Stoffe. Vorreiter dieser Welle waren Frank Castorf und sein Dramaturg Carl Hegemann, beide beriefen sich auf Jacques Derrida. Teile des Publikums reagierten anfangs verstört, aber allmählich entdeckten die Besucher, dass die überbordenden, oft auch wirr anmutenden Abende nichts Anderes waren als Zerrbilder unseres hoch komplexen Alltags. Hinzu kam bei Castorf die subversive Ideologiekritik, ganz besonders stark ausgeprägt in seinem Bayreuther Ring des Nibelungen. Die Dekonstruktion floriert als Stilmittel weiter.

Sehr beliebt sind derzeit auch Rechercheprojekte und Stückentwicklungen. Dabei werden Theaterensembles oder Dramaturgie-Kollektive selbst zu Autoren. Sie widmen sich aktuellen gesellschaftlichen Fragen wie der Flüchtlingskrise. Dass sie dabei auf die Mittel des Dokumentartheaters aus den 1960er und 1970er Jahren zurückgreifen, ist manchen gar nicht so recht bewusst.

Auch das gerade sehr gehypte „Site Specific Theatre“ ist eigentlich ein alter Hut. Denn Theaterensembles haben schon immer gerne an ungewöhnlichen Orten gespielt. Die Berliner Schaubühne ist in den 1970er Jahren mit Hölderlin ins Berliner Olympiastadion gezogen, später mit Romeo und Julia in ein Parkhaus.

Sehr beliebt ist gerade auch die Beteiligung von Laien in professionellen Theatern, etwa bei der Bürgerbühne des Nationaltheaters Mannheim. Das ist ein Modell der Partizipation. Oberammergau macht es seit Jahrhunderten vor. Aber ich muss gestehen: Dem spielerischen Dilettantismus kann ich als Kritiker selten etwas abgewinnen. Den Seelen-Striptease vor Publikum beherrschen professionelle Schauspieler meist sehr viel besser. Wenn aber die Laien von der international gefragten Performance-Gruppe Rimini Protokoll angeleitet werden und sich dabei in „Experten des Alltags“ verwandeln, kommen hochspannende Theaterabende zustande.

Welche Moden gibt es noch? Zu erwähnen wären die multiethnischen Ensembles wie im Berliner Maxim Gorki Theater. Dessen postmigrantisches Theater reflektiert den Assimilationsprozess in der zweiten und dritten Migrantengeneration. Das Gorki-Theater feiert damit enorme Erfolge und wird von Festival zu Festival eingeladen. Das Staatstheater Stuttgart hat kürzlich auch ein multiethnisches Ensemble etabliert.

Soweit, so vielfältig. Das Schauspiel wird sich kontinuierlich weiterentwickeln, es bezieht spartenübergreifend den Tanz, den Film oder Livevideos mit ein und wird so immer mehr zum Gesamtkunstwerk, wie es von Richard Wagner definiert wurde. Dass inzwischen bei Performance-Formaten sogar museale und theatrale Darstellungsformen 
miteinander verschmelzen, hätte sich Wagner aber wohl kaum träumen lassen. Namen wie Christof Schlingensief oder Marina Abramovic stehen für diese Entwicklung.

\section{Literatur}

BRECHT Bertolt (1997): Gedichte I. Bd. 3 der Ausgewählten Werke in sechs Bänden. Frankfurt am Main: Suhrkamp.

DürRENMATT Friedrich (1986): Kritik. Bd. 25 der Werkausgabe. Zürich: Diogenes.

FonTANE Theodor (1979): Theaterkritiken (in vier Bänden). Hg. v. Siegmar GendT, neu eingerichtet v. Andreas Catsch u. Helmuth Nürnberger. München: Ullstein.

KoHsE Petra (1998): Gleiche Stelle - Gleiche Welle - Friedrich Luft und seine Zeit. Berlin: Aufbau.

KerR Alfred (1972): Theaterkritiken. Stuttgart: Reclam.

Kerr Alfred (1985): Mit Schleuder und Harfe - Theaterkritiken aus drei Jahrzehnten. Hg. v. Hugo FetTing. München: Deutscher Taschenbuch Verlag.

Kerr Alfred (1998): „Ich sage, was zu sagen ist“ - Theaterkritiken 1893-1919. Werke in Einzelbänden, Bd. VII, 1. Hg. von Günther RüHLE. Frankfurt am Main: S. Fischer.

Luft Friedrich (2018): Über die Berliner Luft. Feuilletons. Versammelt und mit einem Nachwort versehen von Wilfried F. Schoeller. Berlin: Die Andere Bibliothek.

OelLers Norbert (1979): Ludwig Börne. In: Benno von WIese (Hg.): Deutsche Dichter des 19. Jahrhunderts. Berlin: Erich Schmidt Verlag.

Rost Wolfgang E. (2013): Örtlichkeit und Schauplatz in Fontanes Werken. Berlin: de Gruyter.

Vietor-EngläNDER Deborah (2016): Alfred Kerr - Die Biographie. Reinbek bei Hamburg: Rowohlt.

Zusammenfassung: Kritiker werden häufig karikiert: auf der Bühne, im Film und natürlich in gezeichneter Form. Ein frühes Beispiel bilden Shakespeares ZuschauerKommentare im Sommernachtstraum. In Arsen und Spitzenhäubchen oder dem Animationsfilm Ratatouille findet man ebenfalls Karikaturen. Die Behauptung, die Theaterkritik existiere erst seit Lessings Hamburgischer Dramaturgie, muss zurückgewiesen werden. Ohne Kritik sind weder die antiken Dramatiker-Wettbewerbe noch die Poetik des Aristoteles denkbar. Rein stilistisch betrachtet sind Theaterkritiken von einem starken mündlichen Duktus geprägt, speziell bei Ludwig Börne, Theodor Fontane, Alfred Kerr und Friedrich Luft. Kerrs Behauptung, die Kritik stünde als vierte Gattung neben der Lyrik, Dramatik und Epik diente der Selbststilisierung, andere Kritiker teilen diese Auffassung nicht. Im medialen Kontext stellen sich für heutige Theaterkritiker ständig neue Herausforderungen dar: Zum einen ist der Aktualitätsdruck durch die OnlineKonkurrenz stark gestiegen, zum anderen verlangt die formale Vielfalt (postdramatisches Theater, postmigrantisches Theater, Site Specific Theatre, Rechercheprojekte, Dekonstruktion überlieferter Stoffe etc.) ständig neue Positionsbestimmungen. 
Schlüsselwörter: Theaterkritik, Karikatur, Aktualität, Ludwig Börne, Theodor Fontane, Alfred Kerr, Friedrich Luft

\section{Między Hamletem, Hanswurstem, a terminem oddania tekstu do druku do 22.30 - codzienność krytyki teatralnej}

Streszczenie: Krytycy, skarykaturowani na scenie, w filmie czy też na rysunkach, nie należą do rzadkości. Za jeden z pierwszych przykładów takich karykaturalnych wyobrażeń mogą posłużyć komentarze widzów w Szekspirowskim Snu nocy letniej. Karykatur nie brak także w filmie Arszenik $i$ stare koronki czy też animacji Ratatuj. Twierdzenie, iż krytykę teatralną zapoczątkowała dopiero publikacja Dramaturgii hamburskiej Lessinga, nie znajduje swojego uzasadnienia. Bez krytyki nie do pomyślenia są ani starożytne konkursy dramatopisarskie, ani Poetyka Arystotelesa. Pod względem czysto stylistycznym krytyki teatralne noszą silny ślad oralności, szczególnie u Ludwiga Börnego, Theodora Fontanego, Alfreda Kerra i Friedricha Lufta. Twierdzenie Kerra, że krytyka, obok liryki, dramatu i epiki, to czwarty z podstawowych rodzajów literackich, miało cel autokreacyjny. Inni krytycy nie podzielali jego opinii. Kontekst medialny stawia przed dzisiejszymi krytykami teatralnymi wciąż nowe wyzwania: Internet powoduje wzrost konkurencji i presję aktualności; także różnorodność formalna (teatr postdramatyczny, teatr postmigrancki, Site Specific Theatre, kwerendy w teatrze, dekonstrukcja tradycyjnych tematów etc.) wymaga od krytyka, aby wciąż na nowo określał swoją pozycję.

Słowa kluczowe: krytyka teatralna, karykatura, aktualność, Ludwig Börne, Theodor Fontane, Alfred Kerr, Friedrich Luft

Between Hamlet, Hanswurst and the 10:30 p.m. text submission deadline - the everyday life of theatre criticism

Summary: Critics are often caricatured on stage, in movies and in drawings. One of the first examples are the viewer comments in Shakespeare's A Midsummer Night's Dream. Caricatures are also found in Arsen und Spitzenhäubchen [Arsenic and Old Lace] and the computer-animated film Ratatouille. The claim that theatre criticism began with the publication of Lessing's Hamburgischer Dramaturgie must be rejected. Without theatre criticism, neither the ancient playwright competitions nor Aristotle's Poetics are thinkable. From a purely stylistic perspective, theatre criticism is very heavily inclined towards orality, especially with Ludwig Börne, Theodor Fontane, Alfred Kerr and Friedrich Luft. Kerr's claim that criticism should be considered the fourth major literary genre next to epic, drama, and poetry, served the purpose of self-establishment; other critics do not share this point of view. In the context of media, contemporary theatre critics are faced with new challenges: on the one hand, the pressure to be up to date has risen sharply as a result of online competition, on the other hand the formal diversity (post-dramatic theatre, post-migrant theatre, site-specific theatre, research projects, deconstruction of traditional themes, etc.) constantly demands new positions to be taken.

Keywords: theatre criticism, caricature, up-to-dateness, Ludwig Börne, Theodor Fontane, Alfred Kerr, Friedrich Luft 
Volker Oesterreich Dr. phil., geb. 1960 in Heidelberg, Studium der Germanistik, Amerikanistik und Publizistik an der Freien Universität Berlin. 1987-2001 Kultur-Redakteur der Berliner Morgenpost, von 1990-1994 gleichzeitig freier Hörfunk-Reporter beim RIAS und bei DeutschlandRadio Kultur. Seit 2001 Feuilleton-Chef der RheinNeckar-Zeitung in Heidelberg, außerdem Mitarbeiter des Fachmagazins Die deutsche Bühne. Diverse Jury-Mitgliedschaften. 2007 Journalist Fellow der Stadt Los Angeles. Seit 2014 Gastdozent des Germanistischen Seminars der Universität Heidelberg. Autor und Herausgeber zahlr. Reisebücher, Bildbände und Anthologien, zuletzt erschien der gemeinsam mit seiner Frau Carmen herausgegebene Band 100 Heidelberger Meisterwerke (Ubstadt-Weiher, 2017).

Volker Oesterreich, dr nauk hum., - urodzony w 1960 roku w Heidelbergu, studiował germanistykę, amerykanistykę i publicystykę na Wolnym Uniwersytecie w Berlinie. W latach 1987-2001 pełnił funkcję redaktora działu kultury w dzienniku Berliner Morgenpost, od roku 1990 do 1994 reportażysta w rozgłośni RIAS oraz DeutschlandRadio. Od 2001 roku szef działu kultury heidelberskiej Rhein-Neckar-Zeitung, ponadto współpracownik magazynu Die deutsche Bühne. Juror wielu konkursów. W 2007 roku pełnił funkcję Journalist Fellow miasta Los Angeles. Od roku 2014 docent wizytujący w Katedrze Germanistyki Uniwersytetu w Heidelbergu. Autor i wydawca licznych książek podróżniczych, albumów i antologii. Ostatnio ukazał się wydany wraz z żoną Carmen tom pt. 100 Heidelberger Meisterwerke (Ubstadt-Weiher, 2017).

Volker Oesterreich, PhD, born in 1960 in Heidelberg, studied German and American Philology, as well as journalism at the Free University of Berlin. Between 1987 and 2001, he was the editor at the culture department of Berliner Morgenpost, in the years 1990-1994 he was additionally active as a radio reporter for RIAS and DeutschlandRadio Kultur. Since 2001 he is the head of the features section at Rhein-Neckar Zeitung in Heidelberg and cooperates with Die deutsche Bühne magazine. He sat on the panel of jurors of multiple competitions. In 2007 he acted Journalist Fellow for the city of Los Angeles. Since 2014 he has been Guest Associate Professor of the German Studies Seminar at the University of Heidelberg. He also wrote and edited many travel books, illustrated books and anthologies, such as the recently published volume, edited together with his wife Carmen, 100 Heidelberger Meisterwerke (Ubstadt-Weiher, 2017). 